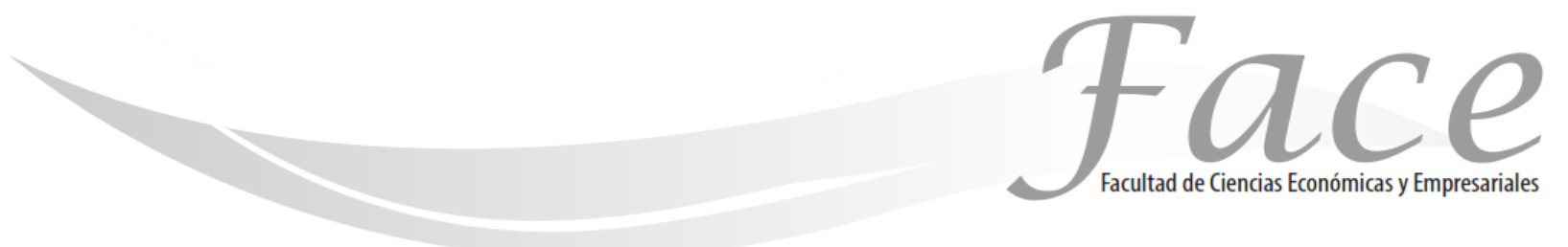

ISSN Impreso: 1794-9920

ISSN Electrónico: 2500-9338

Volumen $19-\mathrm{N}^{\circ} 1$

Año 2019

Págs. $98-108$

\title{
EL IMPACTO DE LAS TIC EN EL MILLENNIAL: MÉXICO-BRASIL-COLOMBIA
}

\author{
Dr. Emigdio Larios Gómez * \\ ORCID: https://orcid.org/0000-0002-3514-1319 \\ Dr. Antonio Carlos Giuliani** \\ ORCID: https://orcid.org/0000-0002-9867-8164 \\ Dr. Thel Augusto Monteiro** \\ ORCID: https://orcid.org/0000-0002-0290-1676
}

Fecha de Recepción: Febrero 19 de 2019

Fecha de Aprobación: Mayo 10 de 2019

\section{Resumen:}

Se trata de una investigación mixta (con enfoque exploratorio-cualitativo y concluyente-cuantitativo), para lograr abarcar varios aspectos importantes en el análisis comparativo del comportamiento del consumidor mexicano, colombiano y brasileño. Para la investigación exploratoria, se usaron los resultados de una investigación realizada en México sobre el nuevo perfil de consumidor mexicano y la revisión literaria de una investigación realizada en Brasil y en Colombia, sobre los diferentes comportamientos generacionales. Se han comparado los datos recabados, usando la técnica de análisis de contenido detonado el contenido manifiesto de los factores en el comportamiento del consumidor como 1) culturales, 2) sociales, 3) personales y 4) psicológicas. En la investigación concluyente, se realizó una encuesta online (a través de formularios de Google Drive y uso de redes sociales como Facebook y Twitter), fue un muestreo no probabilístico por cuotas, siendo todos los encuestados estudiantes de entre 18 y 32 años de edad. La muestra estuvo compuesta por 150 estudiantes (50 mexicanos, 50 colombianos y 50 brasileños). Como resultado principal, se tiene que no existe gran diferencia entre las características del consumidor millennial mexicano, brasileño y colombiano, estos presentan una insensibilidad baja en la comunicación con sus amistades y amigos por el uso de nuevas tecnologías como el internet, redes sociales y comunicaciones móviles. Al igual, las relaciones entre jóvenes de la generación milenio, se ha disminuido de forma cara a cara, prefiriendo la comunicación virtual y el lenguaje pictórico en las plataformas de comunicación. Y gracias al acceso ilimitado a la información (virtuales), la generación $Y$ ha desarrollado una forma de pensar no lineal y multi-tarea, que le ha generado una ansiedad y necesidad de individualizarse (no tiene problemas en cultivar relaciones a corto plazo, así como cambiar de empleo varias veces).

Palabras clave: Capacidades Dinámicas, Mercadotecnia Digital, Desempeño, México

* Dr. en Ciencias Administrativas por el Instituto Politécnico Nacional (IPN). Profesor-Investigador en la Benemérita Universidad Autónoma de Puebla (BUAP).

Contacto: herr.larios@gmail.com

** Dr. en Marketing - Universidad de Sevilla. Diretor Executivo FUMEP - Professor FGV - IBE - área de Marketing Master Coach - Mentor e Analista Comportamental Consultor y docente en posgrado en Colombia, Mexico. Argentina, Bolivia y Brasil. Contacto: giuliani.marketing@uol.com.br

*** Dr.. en Administración de la Universidad Metodista de Piracicaba - UNIMEP. Brasil. Contacto: giuliani.marketing@uol.com.br 


\title{
DYNAMIC CAPACITIES OF MARKETING, DIGITAL MARKETING AND PERFORMANCE IN MEXICAN MIPYMES.
}

\begin{abstract}
:
This is a mixed research (with exploratory-qualitative and conclusive-quantitative approach), to achieve several important aspects in the comparative analysis of the behavior of Mexican, Colombian and Brazilian consumers. For the exploratory research, the results of an investigation carried out in Mexico on the new Mexican consumer profile and the literary review of a research carried out in Brazil and Colombia on the different generational behaviors were used. We have compared the data collected, using the content analysis technique detonated the manifest content of the factors in consumer behavior such as 1) cultural, 2) social, 3) personal and 4) psychological. In the conclusive investigation, an online survey was carried out (through Google Drive forms and use of social networks such as Facebook and Twitter), it was a non-probabilistic sampling by quotas, with all respondents being students between 18 and 32 years of age. The sample consisted of 150 students (50 Mexicans, 50 Colombians and 50 Brazilians). As a main result, there is no great difference between the characteristics of Mexican, Brazilian and Colombian millennial consumers, who present a low insensitivity in communication with their friends and friends through the use of new technologies such as the Internet, social networks and communications. mobile Likewise, relations between young people of the millennial generation have been reduced face to face, preferring virtual communication and pictorial language in communication platforms. And thanks to the unlimited access to information (virtual), generation $Y$ has developed a non-linear and multi-task way of thinking, which has generated an anxiety and need to individualize (has no problems in cultivating short-term relationships, as well how to change jobs several times).
\end{abstract}

Keywords: Millennials; Dehumanization; Technology; Desensitization

\section{CAPACIDADES DINÂMICAS DE MARKETING, MARKETING DIGITAL E DESEMPENHO EM MIPÍMETOS MEXICANOS.}

\section{Resumo:}

Trata-se de uma pesquisa mista (com abordagem exploratório-qualitativa e quantitativa-conclusiva), para alcançar vários aspectos importantes na análise comparativa do comportamento dos consumidores mexicano, colombiano e brasileiro. Para a pesquisa exploratória, foram utilizados os resultados de uma pesquisa realizada no México sobre o novo perfil do consumidor mexicano e a revisão literária de uma pesquisa realizada no Brasil e na Colômbia sobre os diferentes comportamentos geracionais. Comparamos os dados coletados, utilizando a técnica de análise de conteúdo detonou o conteúdo manifesto dos fatores no comportamento do consumidor como: 1) cultural, 2) social, 3) pessoal e 4) psicológico. Na investigação conclusiva, foi realizada uma pesquisa online (através de formulários do Google Drive e uso de redes sociais como Facebook e Twitter), foi uma amostragem não probabilística por cotas, sendo todos os respondentes estudantes entre 18 e 32 anos de idade. . A amostra foi composta por 150 estudantes (50 mexicanos, 50 colombianos e 50 brasileiros). Como resultado principal, não há grande diferença entre as características dos consumidores milenares mexicanos, brasileiros e colombianos, que apresentam baixa insensibilidade na comunicação com seus amigos e amigos por meio do uso de novas tecnologias, como a Internet, as redes sociais e as comunicações. mobile Da mesma forma, as relações entre os jovens da geração do milênio foram reduzidas face a face, preferindo a comunicação virtual e a linguagem pictórica nas plataformas de comunicação. $E$ graças ao acesso ilimitado à informação (virtual), a geração $Y$ desenvolveu um modo de pensar não-linear e multi-tarefa, que gerou uma ansiedade e necessidade de individualizar (não tem problemas em cultivar relacionamentos de curto prazo, bem como mudar de emprego várias vezes).

Palavras-chave: Millennials; Desumanização; Tecnologia; Dessensibilização 
ISSN: 1794-9920 Impreso / Electrónico 2500-9338

Enero - Julio de 2019 - Volumen 19 Número 2, Año 2019 Págs. 98 - 108

\section{INTRODUCCIÓN:}

Una generación es habitualmente definida a través de las experiencias compartidas, dentro de un lapso de tiempo, que hace que las personas adjuntas a este grupo desarrollen valores y conductas compartidas, es decir una historia en común. Más allá del nivel socio económico y de tipo de familia a la que pertenece un mexicano o un brasileño, existe otra segmentación para el análisis de comportamientos de consumo, basada en la vivencia histórica y su impacto socio-cultural, es decir la edad 0 generación a la que pertenecen las personas. Para algunos existen grandes diferencias y para otros existen similitudes entre los miembros de cada generación. Para quienes investigan los perfiles de las generaciones como la $\mathrm{Y}$, la $\mathrm{X}$ y los baby boomers no siempre presentan exactamente los mismos períodos para identificar el nacimiento de los integrantes de cada uno de los grupos. Algunos no diferencian significativamente una de otra generación, sin embargo, en la descripción de las características de las personas que componen estos grupos de personas 0 generaciones (Coimbra y Schikmann, 2001; Maldonado, 2008; Maniero y Sulivan, 2006; Tulgan, 2006), se pueden identificar cinco generación: Tradicionalistas (nacidas entre los años 1926 a 1946), Baby Boomers (nacidas entre los años 1946 a 1964), Generación X (nacidas entre los años 1964 a 1980), Generación Y o Millennials (nacidas entre los años 1980 a 2000) y Generación Z o Nativos Digitales (nacidos a partir del 2000 al 2010) (Oblinger, 2003) (Howe y Strauss, 2007) (Cuesta, 2008).

\section{TECNOLOGÍA Y RELACIONES SOCIALES}

La forma de relacionarnos y realizar la interacción social ha cambiado a medida que los avances tecnológicos, principalmente en las generaciones jóvenes, como la Generación X, la Generación $Y$ y los más recientes la Generación Z (y ya se habla de una generación Alfa). Las nuevas tecnologías han proporcionado nuevos métodos de contacto, pasando del contacto en persona, la escritura de cartas, el telégrafo y el teléfono, hasta los más recientemente usados como las plataformas tecnológicas que van desde modos individualizados, grupales y comunitarios, ya sea a través de accesos a internet por PC's y teléfonos celulares. Con sus respectivas aplicaciones como las videollamadas, mensajes instantáneso, redes sociales, apps y web apps. Pero sabemos muy poco acerca de cómo las diferentes formas de comunicación influyen en las relaciones sociales, la salud y el bienestar en las generaciones pasadas y muy recientes.

El presente trabajo, pretende abordar una perspectiva sobre que las relaciones sociales ahora se experimentan de nuevas maneras debido a los desarrollos tecnológicos.
Y se debe tomar en cuenta que cada vez más los adultos mayores (Babyboomers) utilizan las redes sociales, en comparación con más del $90 \%$ de los jóvenes están en línea y tienen teléfonos celulares, más de la mitad de los adultos mayores de 65 años están en línea y el $78 \%$ posee un teléfono celular (Anderson, 2015; Zickuhr y Madden, 2012). Hermida y Carrasco (2015), argumentan que existe el riesgo de que la tecnología deshumanice a los jóvenes, u que se debe prestar atención a las consecuencias macrosociológicas que esto ocasiona, repercutiendo a efectos humanizadores o no en las relaciones sociales y no es reciente, ya que la revolución tecnológica se dio desde el siglo pasado: "El riesgo de que el avance científicotecnológico quede separado de los avances sociales y humanos se viene trabajando, sistemáticamente desde finales del siglo XIX, sobre todo con las aportaciones iniciales de la Escuela de Frankfurt" (pag. 34).

\subsection{PROBLEMA.}

La aparición de las nuevas tecnologías y su rápida divulgación en las últimas décadas han modificado la forma de vida, incitando grandes impactos en la mayoría de los hábitos de consumo. En la actualidad la generación Millennial ha marcado fuertemente los cambios que se forjan entre una generación y otra, el uso de las nuevas tecnologías es una de las principales razones por las que la generación Millennial se diferencia de sus predecesores, ya que su capacidad de adaptación, intereses y estilo de vida son completamente diferentes.

La generación Millennial (nacidos de los 80 's y 90 's) es la primera en denominarse nativa digital, ya que el mundo cibernético está siempre presente en su vida, se mueven de forma fluida y rápida dentro de las diferentes plataformas y dispositivos que existen, lamentablemente, el exceso de información que recibe esta generación (y sus sucesoras), así como el tiempo que gastan dentro de las redes sociales, hace que el uso de las tecnologías se vea obsoleta y limitada. En la actualidad, las nuevas tecnologías de la información y la comunicación se han integrado en nuestra sociedad de una forma muy rápida, provocando un cambio importante en el estilo de vida actual de las personas y en su forma de interactuar, ya que han revolucionado la forma de difundir la información, provocando importantes cambios, sobre todo en las actividades de ocio, probablemente la influencia más llamativa de las nuevas tecnologías se ha producido entre la población más joven. Si bien al comienzo de la aparición de Internet muchos alertaron de los peligros sobre los más jóvenes (Dibbell (1996), Van Gelder (1996) y Rheingold (1993), el aislamiento social, vulneración del derecho a la intimidad, la manipulación ideológica, etc. las encuestas realizadas al respecto lo niegan (Castells, M., 2002), los jóvenes están demostrando todo lo contrario; ya que no solo gracias al internet mantienen un contacto permanente con sus amigos, conocen y establecen relaciones de 
amistad con personas con sus mismos intereses y aficiones, sino también amplían sus conocimientos y formación.

En definitiva, las nuevas tecnologías permiten a los jóvenes reforzar y ampliar su campo de interacción social. Junto a estos beneficios, también existen estudios que alertan de los riesgos derivados de la excesiva presencia de las nuevas tecnologías en el estilo de vida de esta generación. El primer foco rojo proviene del tiempo total diario dedicado al uso de los aparatos convencionales (televisión, computadora y celular) que, según los datos disponibles, podría superar las 10 horas diarias, es decir, poco menos de la mitad del día (Echeburúa, Labrador y Becoña,2009). Una consecuencia evidente de una dedicación tan amplia del tiempo diario a estas nuevas tecnologías es la disminución del tiempo dedicado a realizar otras actividades que antes eran mucho más habituales (leer, estudiar, salir a pasear, conversar...). Probablemente, el mayor riesgo del uso excesivo de las nuevas tecnologías es el riesgo de generar un comportamiento adictivo que lleve no solo a una dedicación excesiva (lo que puede apartar al chico o chica de otro tipo de actividades más saludables y muy necesarias a ciertas edades), sino a una verdadera dependencia y falta de control sobre sus conductas. Esta adicción es más probable que se genere en el adolescente que en el adulto debido a que su corteza prefrontal se encuentra aún inmadura y su autocontrol no ha alcanzado aún el nivel adulto (Oliva, 2007; Echeburúa y De Corral, 2009).

El uso de la tecnología en la generación actual, la Millennial se ha convertido en una necesidad más que un lujo, pero, ¿Hasta qué punto es aceptable el uso de la tecnología?; ¿Es provechoso el tiempo en que utilizamos la tecnología?; ¿Es posible hablar de una deshumanización o pérdida de la sensibilización por el uso excesivo de la tecnología? En la actualidad podemos observar que gran parte de la población mundial usa la tecnología para hacer de su vida un lugar más cómodo y simplificado.

\section{MATERIALES Y MÉTODOS:}

En esta investigación se propuso profundizar en el comportamiento de la generación Millennial frente a las nuevas tecnologías y su adaptación. El objetivo de esta investigación es analizar el comportamiento de la generación Millennial latinoamericana, respecto al uso de las TIC's en cuanto a su vida diaria, y el impacto que ha generado en su comportamiento en los últimos 3 años, las ventajas que tienen respecto a la generación anterior y posibles consecuencias que se generen por el uso excesivo de las mismas.
Se realizó una investigación mixta (con enfoque exploratorio-cualitativo y concluyente-cuantitativo), para lograr abarcar varios aspectos importantes en el análisis comparativo del comportamiento del consumidor mexicano, colombiano y brasileño. En la primera etapa, la exploratoria-cualitativa se tomó como insumo de información en las categorías de análisis, se usaron los resultados de una investigación realizada en México ${ }^{1}$ sobre el nuevo perfil de consumidor mexicano y la revisión literaria de una investigación realizada en Brasil $^{2}$ y en Colombia $^{3}$ sobre los diferentes comportamientos generacionales. Se han comparado los datos recabados, usando la técnica de análisis de contenido (Bardin, 1996; Mayring, 2000; Pérez, 1994; Krippendorff, 1990), detonado el contenido manifiesto de los factores en el comportamiento del consumidor, en una mezcla de modelos sobre estudios del comportamiento del consumidor de Solomon, Russell-Bennett, y Previte (2013) y Kotler (2012). Los cuales fueron 1) culturales (cultura, subcultura y clase social), 2) sociales (grupos de referencia, familia, roles y estatus), 3) personales (edad y etapa en el ciclo de vida, ocupación, situación económica, estilo de vida personalidad y auto-concepto) y 4) psicológicas (motivación, percepción, aprendizaje, creencias y actitudes).

La segunda etapa, se realizó con la ayuda de un grupo de jóvenes investigadores México-Brasil-Colombia, se aplicó una encuesta online (a través de formularios de Google Drive y uso de redes sociales como Facebook y Twitter) un muestreo no probabilístico por cuotas, en el que se tomó como población a los países de Brasil, Colombia y México (siendo estos los países más desarrollados en cuanto a TIC's), siendo todos los encuestados estudiantes de entre 18 y 32 años de edad. La muestra estuvo compuesta por 150 estudiantes (50 mexicanos, 50 colombianos y 50 brasileños). Se empleó un instrumento adaptado de la propuesta realizada por Labrador1, Villadangos, Crespo y Becoña (2013) con 3 variables 1) Uso de Internet, 2) Uso

\footnotetext{
1 Se trató de una investigación exploratoria-cualitativa, a través de la técnica de análisis de contenido cualitativo (Bardin, 1996; Mayring, 2000; Pérez, 1994; Krippendorff, 1990) y tomando como base o tópicos de estudio los factores en el comportamiento del consumidor como culturales (cultura, subcultura y clase social), sociales (grupos de referencia, familia, roles y estatus), personales (edad y etapa en el ciclo de vida, ocupación, situación económica, estilo de vida personalidad y autoconcepto) y psicológicas (motivación, percepción, aprendizaje, creencias y actitudes) (Solomon, Russell-Bennett, y Previte, 2013) (Kotler, 2012).

2 Se tomaron los datos de la investigación Percepção sobre carreiras inteligentes: diferenças entre as gerações $\mathrm{y}, \mathrm{x}$ e baby boomersPerception on intelligent careers: differences among $y, x$ generations and baby boomers realizada por Elza Fátima RosaVelosoaJoel SouzaDutrabLina EikoNakatac en Brasil, 2016. En REGE-Revista de Gestão, 23(2), 88-98. ${ }^{3}$ Se tomaron los datos de la investigación Posada López, M. C., \& White Arbeláez, M. (2016). Tipificación del consumo de la generación" Millennial" en Medellín en los estratos 4, 5 y 6 (Doctoral dissertation, Universidad EIA).
} 
de videojuegos y 3) Uso del celular; integrado por 22 preguntas colocadas en escala de Likert, validado por expertos (integrantes del Comité Científico InterInstitucional México-Brasil-Colombia), como se muestra en la Tabla 1, correspondiente a la operación de variables.

Tabla 1. Operación de Variables.

\begin{tabular}{|c|c|c|}
\hline Ítem & Variable & Descripción \\
\hline 1 & \multirow{9}{*}{$\begin{array}{l}\text { Uso de } \\
\text { Internet }\end{array}$} & Me conecto diariamente a Internet \\
\hline 2 & & Me conecto más de 10 horas a la semana \\
\hline 3 & & Me conecto más tiempo del que en un principio había pensado estar \\
\hline 4 & & $\begin{array}{l}\text { Me conecto a internet y/o el correo, no por motivos laborales, de ocio o } \\
\text { escolares, sino por la necesidad de conectarme }\end{array}$ \\
\hline 5 & & He notado que cada vez estoy más tiempo conectado \\
\hline 6 & & $\begin{array}{l}\text { He dejado de hacer actividades que antes me gustaban por estar } \\
\text { conectado }\end{array}$ \\
\hline 7 & & $\begin{array}{l}\text { He intentado alguna vez disminuir el tiempo que dedico a Internet, sin } \\
\text { conseguirlo }\end{array}$ \\
\hline 8 & & Me pongo de mal humor si no puedo conectarme \\
\hline 9 & & $\begin{array}{l}\text { Cuando no estoy conectado, a menudo pienso que estará pasando en la } \\
\text { red }\end{array}$ \\
\hline 10 & \multirow{7}{*}{$\begin{array}{l}\text { Uso de } \\
\text { videojuegos }\end{array}$} & Utilizo videojuegos \\
\hline 11 & & Juego cada día \\
\hline 12 & & Juego más de 2 horas al día \\
\hline 13 & & He dejado de hacer actividades que antes me gustaban por jugar \\
\hline 14 & & He intentado disminuir el tiempo que dedico a jugar y no lo he conseguido \\
\hline 15 & & Me pongo de mal humor si no puedo jugar \\
\hline 16 & & Cuando no estoy jugando, pienso a menudo en el videojuego \\
\hline 17 & \multirow{6}{*}{$\begin{array}{l}\text { Uso del } \\
\text { celular }\end{array}$} & Gasto más dinero del que considero necesario en el uso del celular \\
\hline 18 & & Chateo todos los días \\
\hline 19 & & Si chateo todos los días, dedico más de 4 horas \\
\hline 20 & & Me siento intranquilo/a o desprotegido/a si te dejo el móvil en casa \\
\hline 21 & & $\begin{array}{l}\text { He dejado de lado amigos o amigas para dedicarme más a chatear con } \\
\text { mis contactos del móvil }\end{array}$ \\
\hline 22 & & Me he apropiado de dinero que no me pertenece para recargar el celular \\
\hline
\end{tabular}

Fuente: Elaboración propia. 
Bajo esta perspectiva, se han planteado las siguientes, y se describen las relaciones de las variables en el modelo, ver Figura 1, para las hipótesis propuestas:

$H_{1}$ : Existe una relación significativa entre la disminución de la comunicación (familiar) en los jóvenes millennials México-Brasil-Colombia, debido al uso de tecnología en su vida diaria, que los vuelve insensibles.

$H_{2}$ : Existe una relación significativa entre el aumento de la convivencia virtual (amistad) en los jóvenes millennials México-Brasil-Colombia, debido al uso de tecnología en su vida diaria, que los vuelve insensibles.

Figura 1. Modelo hipotético con variables.
Con base en la operacionalización de variables, se ha determinado las siguientes hipótesis:

Hipótesis nula (Ho): $\mu 1=\mu 2=\mu 3$

Las medias de los grupos $\mu 1$ (MM) $\mu 2$ (MB) y $\mu 3$ (MC) son iguales y por tanto las diferencias encontradas pueden explicarse por el azar. Es decir, no hay diferencias entre el impacto de las nuevas tecnologías en las relaciones sociales de los millennials México-Brasil-Colombia, que los convierta en insensibles.

Hipótesis alternativa $(\mathrm{H} 1): \mu 1 \neq \mu 2 \neq \mu 3$

Las medias de los grupos $\mu 1$ (MM $\mu 2$ (MB) y $\mu 3$ (MC) no son iguales, 0 al menos uno de los grupos tiene una media distinta del resto de grupos. Es decir, si hay diferencias diferencias entre el impacto de las nuevas tecnologías en las relaciones sociales de los millennials México-BrasilColombia, que los convierta en insensibles.
Variable
Variables Independiente
1. Uso de internet
2. Uso de videojuegos
3. Uso de celular [
Variable Dependiente

$\left\{\begin{array}{l}\text { Amistad } \\ \text { Familiar }\end{array} \begin{array}{l}\begin{array}{l}\text { Convivencia } \\ \text { (VIRTUAL) }\end{array} \\ \begin{array}{l}\text { Comunicación } \\ \text { (CARA A CARA) }\end{array}\end{array}\right.$

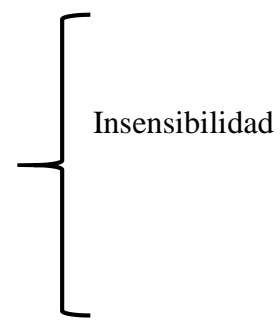

Fuente: Elaboración propia.

Asimismo, se realizó la prueba ANOVA para la comparación de medias de tres grupos clasificados como MM (millennials mexicanos), MB (millennials brasileños) y $\mathrm{MC}$ (millennials colombianos). Bajo un diseño ex post facto retrospectivo (León \& Montero, 2003), que permitió analizar las medias de la variable factor a la motivaciónsatisfacción laboral, como se observa en la Tabla 1, procurando la mayor representatividad de la población de cada grupo (Altman \& Bland, 1996).

\section{RESULTADOS}

\subsection{Resultados Cualitativos.}

A continuación, en la Tabla 2, se describe la comparación entre las características generacionales de los consumidores mexicanos, colombianos y brasileños, obtenidos de la revisión literaria y de análisis de contenido realizado: 
Tabla 2. Resultados

\begin{tabular}{|c|c|c|}
\hline \multicolumn{3}{|c|}{ La Generación Y o Millennials (1980 a 2000) } \\
\hline Perfil del Mexicano & Perfil del Brasileño & Perfil del Colombiano \\
\hline $\begin{array}{l}\text { El millennial a la mexican style- } \\
\text { recibió a temprana edad el } \\
\text { impacto de la caída del muro de } \\
\text { Berlín y el fin de la guerra fría, así } \\
\text { como la reconfiguración de la } \\
\text { geografía mundial. Herederos del } \\
\text { México tercermundista, con el } \\
\text { estreno de la democracia } \\
\text { nacional (por fin se configura el } \\
\text { PRI-PAN-PRD), la preocupación } \\
\text { y apreciación de los recursos } \\
\text { naturales (aire, mar, tierra) y } \\
\text { considerados como la primera } \\
\text { generación nativa digital. Le han } \\
\text { dado como resultado con esto un } \\
\text { optimismo de vida al seguir } \\
\text { viviendo con sus papás, a Google } \\
\text { como un Dios robot-invisible que } \\
\text { venerar y una sólida seguridad }\end{array}$ & $\begin{array}{l}\text { Crecieron en contacto con las } \\
\text { tecnologías de la información y } \\
\text { son más individualistas. Defiende } \\
\text { sus opiniones y prioriza el lado } \\
\text { personal en relación a las } \\
\text { cuestiones profesionales. Cuando } \\
\text { las personas de esa generación } \\
\text { comenzaron a nacer, encontraron } \\
\text { a Brasil pasando por una gran } \\
\text { inestabilidad económica y, poco } \\
\text { después, reinstalando la la } \\
\text { democracia; en el escenario } \\
\text { mundial, la cultura de la } \\
\text { impermanencia y la falta de } \\
\text { garantías, con mercados volátiles. } \\
\text { Este grupo más joven es } \\
\text { equivalente al } 33 \% \text { de la población } \\
\text { económicamente activa. }\end{array}$ & $\begin{array}{l}\text { En Colombia la generación millennial es } \\
\text { un híbrido entre un deseo de construir un } \\
\text { mejor futuro, salir adelante, conectarse y } \\
\text { compartir el contenido permanentemente, } \\
\text { creada por la inequidad económica. } \\
\text { Poseen una personalidad crítica y } \\
\text { avanzada porque han vivido en tiempos de } \\
\text { gran desarrollo tecnológico, económico y } \\
\text { social. La mayoría de ellos vieron a sus } \\
\text { padres trabajar fuertemente para darles } \\
\text { todas las comodidades, beneficios y } \\
\text { oportunidades que los 1980s ofrecía al } \\
\text { mundo, razón por la cual crecieron } \\
\text { escuchando que podían lograr todo lo que } \\
\text { querían en la vida, fueron muy } \\
\text { consentidos por sus padres y crecieron } \\
\text { con la filosofía de disfrutar la vida al } \\
\text { máximo y de hacer a ellos mismos su } \\
\text { principal prioridad de vida. }\end{array}$ \\
\hline
\end{tabular}

para vivir solos, sin hijos 0

divorciados a su corta edad.

Ya son digitales:

- Son multitareas.

- No conciben la realidad sin tecnología.

- La calidad de vida tiene prioridad.

- Son emprendedores.

- Usan más tipos de tecnología para entretenimiento: Internet, SMS, Reproductor de CD, MP3, MP4, DVD, BlueRay, Iphone, entre otros.

- El lujo (para la generación X) son productos "básicos".

- Son creadores y no replicadores.

- Son líderes y seguidores.

- Tienen amigos virtuales en lugar de imaginarios.

- La preferencia sexual es flexible.

- Todavía son sensibles a los acontecimientos que perjudican al ambiente y a las mascotas.

Son criticados por transmitir, fomentar y ser iniciadores de la des-humanización de su generación.

Fuente: Elaboración propia con información de Larios-Gómez y De la Vega (2017); Veloso, Dutra, y Nakata, (2016); y Posada \& White (2016).

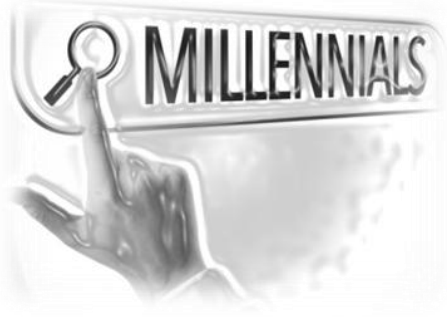

\subsection{Resultados Cuantitativos.}

La fiabilidad de la investigación es adecuada, el coeficiente de Alfa de Cronbach fue de 0.930 en general para México y para la variable uso de internet fue de 0.930 , para uso de video juegos 0.750 y para uso del celular 0.844 . Para Brasil fue de .923 en general y para la variable uso de internet fue de 0.900 , para uso de video juegos 0.804 y para uso del celular 0.812 y inalmente para Colombia fue de .9123 en general y para la variable uso de internet fue de 0.922 , para uso de video juegos 0.844 y para uso del celular 0.899 (ver Tabla 4). 
Tabla 4. Fiabilidad de la investigación.

$\begin{array}{lll}\text { México } & \text { Brasil } & \text { Colombia }\end{array}$

\begin{tabular}{lcccccc}
\hline \multicolumn{1}{c}{ Variables } & $\begin{array}{c}\text { Alpha de } \\
\text { cronbach }\end{array}$ & General & $\begin{array}{c}\text { Alpha de } \\
\text { cronbach }\end{array}$ & General & Alpha de cronbach & General \\
\hline Uso de internet & 0.930 & 0.930 & 0.900 & 0.923 & 0.922 & 0.912 \\
\hline $\begin{array}{l}\text { Uso de } \\
\text { videojuegos }\end{array}$ & 0.75 & & 0.804 & & 0.845 & \\
\hline Uso del celular & 0.844 & & 0.812 & & 0.899
\end{tabular}

Fuente: Elaboración propia.

En los resultados podemos observar que el comportamiento de la muestra respecto al uso de la tecnología es muy parecida entre mexicanos, colombianos y brasileños, ya que al realizar el análisis descriptivo en el programa SPSS 21, los datos no se encuentran tan separados unos de los otros, el promedio de horas de consumo es de 8-9 horas en los estudiantes millennials mexicanos, de 8 a 10 horas para los colombianos y de 8.59.5 horas para los brasileños aproximadamente y la mayoría de la muestra usa algún equipo móvil para conectarse $45 \%, 46 \%$ y $48 \%$ del tiempo para mexicanos, colombianos y brasileños respectivamente, usualmente utilizan el internet para redes sociales, comunicarse con amigos o hacer actividades de la escuela, $67 \%$ en México y $71 \%$ en Colombia y $72 \%$ en Brasil.

En el caso del análisis inferencial, se tomaron en cuenta dos aspectos muy importantes el tiempo que se invierte en la tecnología y el uso que se le da, al realizar la corrida de información, encontramos que a pesar de que la muestra utiliza la tecnología a diario (Dibbell, 1993, 1996), el uso que le dan es principalmente para actividades (Van Gelder,1985-1996), los resultados fueron en el aspecto laboral (México en un 34\%, Colombia 38\% y Brasil en un $45 \%$ ), educativo (México en un $32 \%$, Colombia $33 \%$ y Brasil en un 31\%) o como medio de comunicación ( $89 \%$ en México, $91 \%$ y $93 \%$ en Brasil), con esto podemos decir que la generación Millennial presenta una deshumanización por la influencia de la tecnología, por un aislamiento de los individuos, esto último por decisión propia.
En el análisis de datos, se identificaron correlaciones importantes significantes en la influencia de la tecnología en las relaciones sociales y el comportamiento de los millennials, tanto en mexicanos como en los brasileños. Con base en el objetivo de la presente investigación, se tomaron las 3 correlaciones más importantes en cada país y se compraron entre ellos.

Correlación 1: Tanto para el caso de México -Mx, para Colombia-Co, como para Brasil $-\mathrm{Br}$, existe una relación estrecha ( $M x r=.523$, Co $r=.510$ y $B r r=.499$ ) entre el ítem He dejado de lado amigos o amigas para dedicarme más a chatear con mis contactos del móvil y el ítem He dejado de hacer actividades que antes me gustaban, por jugar en el móvil ( $\mathrm{p}=0.00$, para todos $\mathrm{Mx}$, Co y $\mathrm{Br}$ ). Como se muestra en la Tabla 5, no existe una diferencia contundente entre el impacto de las TIC de los millennials mexicanos, en los millennials colombianos y los millennials brasileños, invierten y prefieren pasar más tiempo con amigos en el celular o móvil, que con los amigos de forma presencial. Sus conversaciones son más de forma remota, aún si se encuentran de manera cercana o no. 
Tabla 4. Correlación 1

He dejado de lado amigos o amigas para He dejado de hacer actividades que antes me dedicarme más a chatear con mis contactos del móvil gustaban, por jugar en el móvil

\begin{tabular}{l|ll}
\hline MÉXICO & Pearson & 0.523 \\
BRASIL & Pearson & 0.499 \\
COLOMBIA & Pearson & 0.510
\end{tabular}

Fuente: Elaboración propia con SPSS 21

Correlación 2: Tanto para el caso de México -Mx, para Colombia -Co, como para Brasil -Br, tampoco existe una relación estrecha ( $M x r=.539$, Co $r=501$ y $B r r=$.521) entre el ítem Cuando no estoy conectado, a menudo pienso que estará pasando en las redes sociales y el ítem Me siento intranquilo/a o desprotegido/a si te dejo el móvil en casa ( $p=0.00$, para todos Mx, Co y Br). Como se muestra en la Tabla 6, no existe una diferencia contundente entre el impacto de las TIC de los millennials mexicanos, y los millennials colombianos y los millennials brasileños, ambos sienten la necesidad de saber que está pasando en las redes sociales y por ello invierten en promedio 9 horas diarias en el internet.

Tabla 5. Correlación 2

Cuando no estoy conectado, a menudo pienso que estará pasando en las redes sociales
Me siento intranquilo/a o desprotegido/a si te dejo el móvil en casa

\begin{tabular}{l|ll}
\hline & & \\
MÉXICO & Pearson & 0.539 \\
BRASIL & Pearson & 0.521 \\
\hline COLOMBIA & Pearson & 0.501
\end{tabular}

Fuente: Elaboración propia con SPSS 21

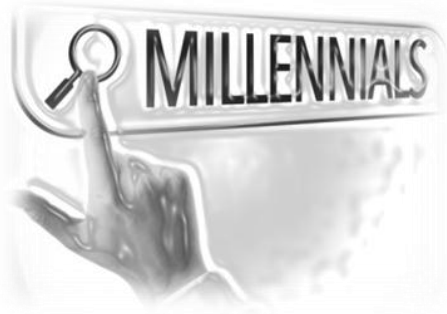

\section{CONCLUSIONES:}

Un panorama general de la investigación nos muestra que la generación Millennial se adapta mejor a las nuevas tecnologías, creando un estilo de vida más cómodo, ya que puede ser usada para un sin número de actividades, desde la búsqueda de un artículo de investigación hasta calentar la comida de un día anterior, la tecnología puede ser nuestra aliada o nuestra peor enemiga, todo depende de nuestra capacidad de adaptación. Cómo podemos observar, no es la tecnología la que deshumaniza, sino las propias personas a través de ella, ya que nosotros mismos somos los que decidimos cuanto tiempo invertimos en los aparatos tecnológicos y el aporte que nos brindan a nuestra vida, el uso de las tecnologías se encuentra en un 
constante cambio y debemos tomar en cuenta que la generación sucesora de la Millennial, ya que está creciendo a pasos agigantados en este rubro, por lo que sería interesante conocer qué camino tomará en unos cuantos años nuestro estilo de vida y la forma de utilizar la tecnología en ella.

Clasificar las generaciones y nombrarlas se configura como un hábito cada vez más común en estudio del comportamiento de consumo y sociales. Ya no basta catalogar a los individuos o a los mercados por lugar, por edad, por sexo o por nivel de ingresos. Ahora vamos más allá de estos, con estilos de vida y el impacto de los factores y premisas externas (sociales, económicas 0 políticas que influyen en su contexto). Es decir, vistos por generaciones integradas por individuos que nacieron en una la misma época viviendo un mismo contexto histórico, político, económico y social el cual determina actitudes y comportamientos, impactando, consecuentemente, en la evolución del individuo hacia la sociedad. No se perciben diferencias entre los comportamientos entre millennials mexicanos y brasileños. Si bien, los Millennials poseen dificultades en relaciones interpersonales, no poseen habilidades humanas en la vida social y con ello, comienza la duda de cómo su relación profesional. También tienen dificultades de relacionarse con el prójimo y mirar en sus ojos por unos instantes para un breve diálogo, contrario a que puede platicar con cualquier extraño y contarle a través de sus redes sociales toda su vida, la cual ni la sabe sus padres. Por lo que se acepta la hipótesis, tanto para México, Colombia y para Brasil: La generación Millennial está cruzando por un proceso de alejamiento social por el uso excesivo de la tecnología. Ha dejado de lado las reuniones presenciales con amigos para dedicarse más a chatear con sus contactos del móvil y ha dejado de hacer actividades que antes le gustaban, por jugar en el móvil. Porque cuando no está conectado, a menudo piensa que estará pasando en las redes sociales y se siente intranquilo o desprotegido si te deja el móvil en casa.

Definitivamente, gracias al acceso ilimitado a la información (medios virtuales), no solo la generación $Y$ ha desarrollado una forma de pensar no lineal y multi-tarea, que le ha generado una ansiedad y necesidad de individualizarse (no tiene problemas en cultivar relaciones a corto plazo, así como cambiar de empleo varias veces). También la Generación X ha sido impactada, en ahora en sus 40's años y sus inicios de los 50's años de vida. Solo nos resta empezar a analizar cómo viene la próxima generación: la Generación Alpha, 2010, quienes son los Nativos Digitales desde su gestación, también denominada la Generación Z (nacidos a partir del 2000). Y son las nuev@s "promesas de México". Son niños y jóvenes que nacieron en el mundo post-internet: los llamados nativos digitales. A diferencia de las generaciones anteriores, "Inmigrantes digitales", la Generación Z es la primera que creció alfabetizada digitalmente y libre de cualquier acento, mayúscula 0 palabra completa para hablar o escribir.

\section{REFERENCIAS:}

Bardin, L. (1996) (2ªed). Análisis de contenido. Madrid: Akal.

Batista, F. H. A. (2014). Grupos geracionais e 0 comprometimento organizacional: um estudo em uma empresa metalúrgica de Caxias do Sul (Doctoral dissertation).

Castells, M., (2001) «The Internet Galaxy. Reflections on the Internet, Business and Society», Oxford University Press.

Cavazotte, F. D. S. C. N., da Costa Lemos, A. H., y de Aquino Viana, M. D. (2012). Novas gerações no mercado de trabalho: expectativas renovadas ou antigos ideais?. Cadernos Ebape. br, 10(1), 162180.

Coimbra, R. G. C., y Schikmann, R. (2001). A gera,cão net. Campinas: Anais Anpad

Dibbell, J., (1993, 1996.) «Taboo, consensus, and the challenges of democracy in an electronic forum», en Kling, R. (ed.), «Computerization and Controversy», págs. 553-568.

Echeburúa, E. y De Corral, P. (2009). Las adicciones con o sin droga: una patología de la libertad. En E. Echeburúa, F. J. Labrador y E. Becoña (Coords). Adicción a las nuevas tecnologías en adolescentes y jóvenes (pp. 29-42). Madrid: Pirámide.

Hermida, O. V., \& Carrasco, B. B. (2015). La implicación de las TIC en la humanización de la sociedad. Opción, 31(78), 31-41.

Howe, N., y Strauss, W. (2007). Millennials Go to College. EE.UU. New York: Vintage Books

Kotler, P. (2012). Dirección de Marketing. México: Pearson.

Krippendorff, K. (1990). Metodología de análisis de contenido. Buenos Aires: Paidós. 
Solomon, M. R., Russell-Bennett, R., y Previte, J. (2013). Consumer behaviour: Buying, having, being. Australia.: Pearson.

The Configuration of A Profile Of The New Consumer In Mexico. Caderno Profissional de Marketing-UNIMEP, 5(2), 93-114.

Labrador, F. J., Villadangos, S. M., Crespo, M., \& Becoña, E. (2013). Desarrollo y validación del cuestionario de uso problemático de nuevas tecnologías (UPNT). Anales de psicología, 29(3), 836-847.

Maldonado, J. A. (2008). Factores que influyen en la conducta del consumidor. Una aproximación desde las ciencias sociales (págs. 21-30). México: COLPARMEX.

Maniero, L. A., y Sulivan, S. E. (2006). The opt-out revolt: why people are leaving companies to create kaleidoscope careers. Mountain View: DaviesBlack Publishing.

Martin, C. A., y Tulgan, B. (2006). Managing the generation mix: From urgency to opportunity (2nd ed.). Amherst: HRD Press.

Mayring, P. (2000). Qualitative Content Analysis. Forum Qualitative Sozialforschung / Forum: Qualitative Social Research, 1(2).

Oblinger, D. (2003). Boomers, Gen-Xers, and Millennials: Understanding. EDUCAUSE Review, vol. 38, no. 4.

Oliva, A. (2007). Desarrollo cerebral y asunción de riesgos durante la adolescencia. Apuntes de Psicología, $25,239-254$.

Rheingold, H., (1993) «The virtual community: Homesteading on the electronic frontier», Addison-Wesley, Reading, MA.

Rodrigues, A. I., y Zatz, F. Z. (2016). A escola contemporânea e 0 diálogo com a Geração Z: Estudo de caso de educomunicação no ensino médio do Colégio Israelita Brasileiro. Educação, Cultura e Comunicação, 7(13).Cuesta, E. M. (2008). EL IMPACTO DE LA GENERACIÓN MILLENNIAL. XV Jornadas de. Buenos Aires: Facultad de Psicología - Universidad de Buenos Aires.

Van Gelder, L., (1985-1996) «The strange case of the electronic lover», en Kling, R., 1996 (ed.), "Computerization and Controversy», $2^{\mathrm{a}}$ edición, págs. 533-546, Academic Press, San Diego, CA.,.

Veloso, E. F. R., Dutra, J. S., y Nakata, L. E. (2016). Percepção sobre carreiras inteligentes: diferenças entre as gerações $y$, xe baby boomers. REGE-Revista de Gestão, 23(2), 88-98. 